\title{
Aortic arch interruption presenting with absence of all limb pulses
}

\author{
G. P. SHARRATt, R. LEANAGE, J. L. MONRO, AND E. A. SHINEBOURNE \\ Wessex Regional Cardiothoracic Centre, Southampton, and Brompton Hospital, London
}

SUMMARY Three patients with aortic arch anomalies presented with severe heart failure, acidaemia, and poor or absent upper and lower limb pulses. Prominent carotid arterial pulsations were detected which distinguished the condition clinically from aortic atresia. In 2 patients with a left aortic arch, interruption was distal to the left common carotid artery and there was an aberrant right subclavian. In the 3rd patient with a right aortic arch, interruption was distal to the right common carotid and there was an aberrant left subclavian artery. By dilating the narrowed ductus, prostaglandin E2 infusion corrected the acidosis. Successful surgical correction was achieved in one patient by direct anastomosis of descending aorta to ascending aorta and the base of the left common carotid artery.

Complete interruption of the aortic arch is a rare congenital anomaly which normally presents in infancy with heart failure and acidaemia (Fishman et al., 1976). There is almost always a ventricular septal defect and persistence of the ductus arteriosus (Roberts et al., 1962). When the interruption is between the left common carotid artery and the left subclavian artery (type B of Celoria and Patton (1959)), the descending aorta is supplied from the pulmonary artery via the ductus arteriosus and also by retrograde flow from the left vertebral artery, which also supplies the left subclavian artery.

When an infant presents with cyanotic congenital heart disease, heart failure, acidaemia, and reduced or absent upper and lower limb pulses the diagnosis of aortic atresia is likely. With type B interruption of the aortic arch, when the right subclavian artery arises aberrantly from the descending aorta, and the ductus arteriosus constricts (Fig. 1), a similar clinical picture is found. In this case, however, there will be normal or bounding carotid pulses. This is now a surgically correctable lesion and hence the importance of its recognition. We report 2 cases that presented in this manner, and a further similar patient with a right-sided aorta, grossly hypoplastic arch distal to the right common carotid, and aberrant left subclavian artery.

Southampton University

G. P. SHARRATT, lecturer in medicine

Wessex Regional Cardiothoracic Centre, Southampton

J. L. MONRO, consultant cardiac surgeon

Brompton Hospital, London

R. LEANAGE, registrar in cardiology

E. A. SHINEBOURNE, consultant paediatric cardiologist

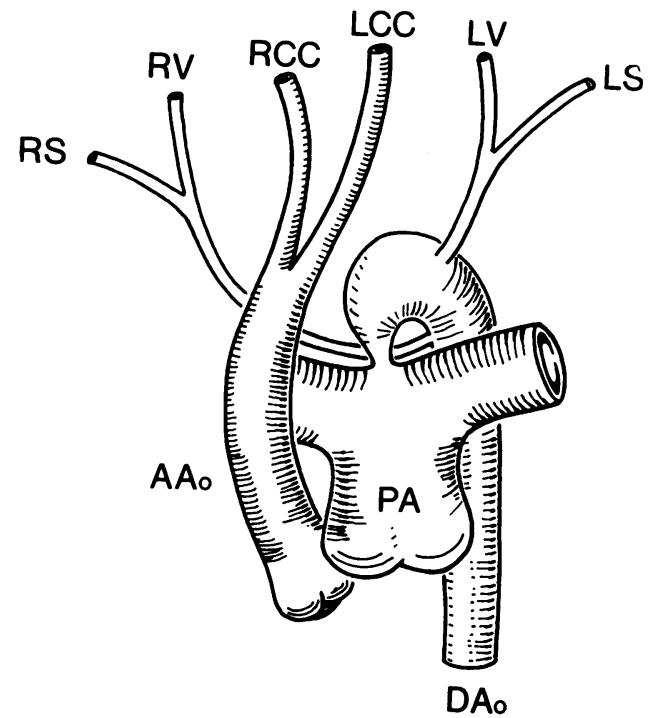

Fig. 1 The ascending aorta (AAo) gives off only the right common carotid artery $(R C C)$ and the left common carotid artery $(L C C)$. The pulmonary artery $(P A)$ connects with the descending aorta (DAo) through a constricted ductus arteriosus. The left subclavian (LS) and right subclavian $(R S)$ arteries arise from the descending aorta and give off the right vertebral (RV) and left vertebral (LV) arteries.

\section{Case reports}

Case 1. A Caucasian baby boy, normal delivery, born at 39 weeks' gestation with a birthweight of 
$2.9 \mathrm{~kg}$. On day 2 a murmur was noted and at that time all the peripheral pulses were normal. The next day he became cyanosed, tachypnoeic, and was found to be in heart failure with no pulses palpable in the arms or legs.

He was transferred to the Wessex Cardiothoracic Centre where he was found to be severely tachypnoeic, sweating profusely, with $3 \mathrm{~cm}$ hepatomegaly. There were no pulses palpable in the limbs but bounding carotid pulses were present bilaterally. There was a much increased parasternal impulse and on auscultation there was an ejection click, soft short ejection systolic murmur, and a single second sound. The ECG suggested right ventricular hypertrophy and the left ventricular potentials were well seen. The chest $x$-ray showed a large heart with plethoric lung fields.

The echocardiogram showed the presence of 2 atrioventricular valves, 2 ventricles and 2 arterial valves, the anterior of which had midsystolic closure suggesting that it was the pulmonary valve.

Cardiac catheterisation proved the diagnosis of complete interruption of the aortic arch between the left common carotid artery and the left subclavian artery with the right subclavian artery arising aberrantly from the descending aorta (Fig. 1). Injection into the ascending aorta showed delayed retrograde filling of both vertebral arteries, and hence both subclavian arteries and the descending aorta. There was also a ventricular septal defect and a persistent ductus arteriosus. When the catheter was passed from the pulmonary artery to the descending aorta there was loss of pressure waveform and injection of contrast showed very slow clearing from the descending aorta. There was also a small left-toright shunt at atrial level which proved to be due to a secundum atrial septal defect.

Surgical correction was performed the same day using profound hypothermia, and total circulatory arrest at $18^{\circ} \mathrm{C}$ (Barratt-Boyes et al., 1971). The technique was virtually the same as that used by Trusler and Izukawa (1975). The descending aorta was dissected and mobilised and the duct divided from the pulmonary artery. The descending aorta, with most of the duct tissue removed, was then anastomosed to the ascending aorta and base of left common carotid artery. The pulmonary arteriotomy was closed and the right atrium incised. The atrial septal defect was closed directly, and the ventricular septal defect was closed through the tricuspid valve using a dacron patch. Total circulatory arrest lasted $68 \mathrm{~min}$ and the baby was taken off the ventilator and extubated 6 hours postoperatively. The postoperative course was complicated by intermittent collapse of the right upper lobe, but he left hospital 16 days after the operation.
He has not yet been reinvestigated but was well and thriving 3 months after surgery although he appears to have developed some aortic narrowing as the right arm and leg pulses are absent; however the left arm pulse is still present, but reduced.

Case 2. A Caucasian baby girl, normal delivery, born at 41 weeks. At 36 hours severe tachypnoea and cyanosis was noted and the baby was transferred to Brompton Hospital. On examination the child was extremely sick with absent limb pulses. Bounding temporal and carotid pulses were noted. Respiratory rate was $90 / \mathrm{min}$. Examination revealed a split second sound with a loud pulmonary component and a grade $3 / 6$ pan systolic murmur at the lower left sternal edge. Arterial $\mathrm{Po}_{2}$ in $100 \%$ oxygen in the right radial artery was $17 \cdot 3 \mathrm{kPa}(130 \mathrm{mmHg}),\left[\mathrm{H}^{+}\right]$ $64 \mathrm{nmol} / 1$ ( $\mathrm{pH} 7 \cdot 19)$, base excess $19 \mathrm{mmol} / 1$. Cardiac catheterisation showed a left-to-right shunt at ventricular level, equal ventricular pressures, and a pulmonary artery pressure of $52 / 27 \mathrm{mmHg}$. The catheter was advanced to the descending aorta where the systolic pressure was $15 \mathrm{~mm}$ lower, indicating constriction of the ductus. Left ventricular cineangiography showed a single high ventricular septal defect, filling of the ascending aorta and both common carotid arteries, but no opacification of the subclavian arteries or descending aorta (Fig. 2).

Injection into the left-sided descending aorta showed the left subclavian artery, the aberrant right subclavian, and the constricting ductus arteriosus (Fig. 3). Attempts at surgical correction were unsuccessful: the child died on the operating table.

The necropsy showed situs solitus, concordant atrioventricular and concordant ventriculo-arterial connections. The left-sided ascending aorta was a small channel giving rise only to right and left carotid arteries, confirming the angiographic diagnosis. The pulmonary artery was in continuity through a ductus with the descending aorta, from which arose the left and then right subclavian arteries. The right subclavian artery was posterior ascending behind the oesophagus. The arch was absent between left common carotid and ductus. The large ventricular septal defect resulted from backward displacement of the parietal insertion of the infundibular septum. The displaced infundibular septum caused severe narrowing of the left ventricular outflow tract, i.e. subvalvar aortic stenosis.

Case 3. A Caucasian baby boy, normal delivery, born at 40 weeks. At 24 hours severe tachypnoea and cyanosis was noted and the baby was admitted to the Brompton Hospital at $\mathbf{4 8}$ hours. The respiratory rate was $80 / \mathrm{min}$. All limb pulses were barely palpable although temporal and carotid pulsations 


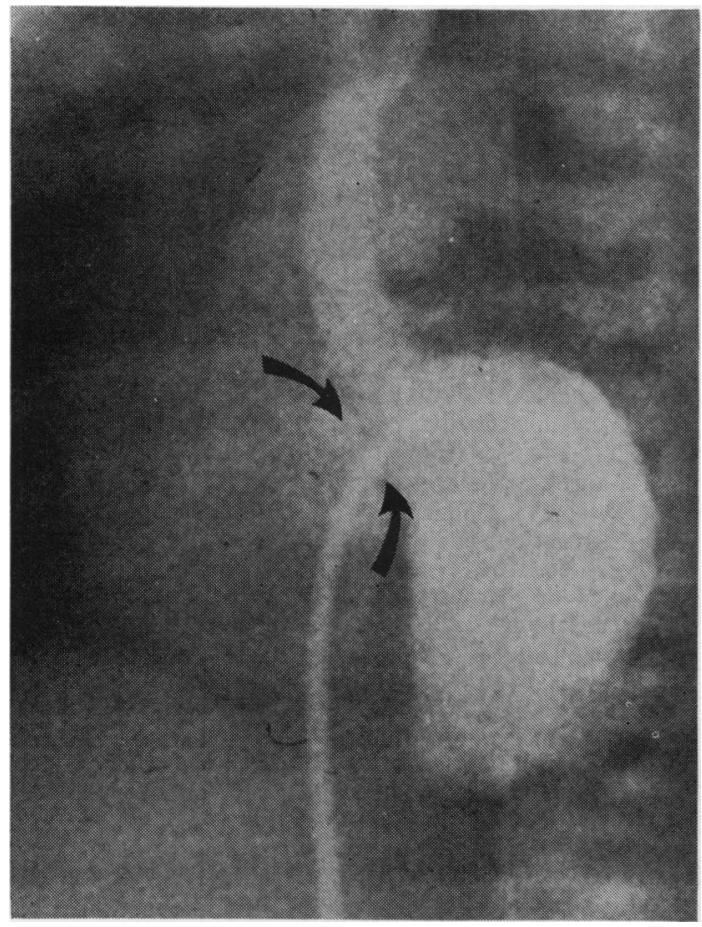

Fig. 2 Left ventricular angiogram, lateral projection. The ascending aorta arises from the left ventricle and gives off the right and left common carotid arteries.

The catheter is across the ventricular septal defect (between arrows) and there is faint left-to-right shunting of contrast across the ventricular septal defect.

were felt. On asucultation there was a split second sound with a loud pulmonary component and a short systolic murmur at the lower left sternal edge. Arterial $\mathrm{Po}_{2}$ in $100 \%$ oxygen was $8 \mathrm{kPa}(60 \mathrm{mmHg})$, $\left[\mathrm{H}^{+}\right] 64 \mathrm{nmol} / 1$ (pH 7.19), base excess $18 \mathrm{mmol} / \mathrm{l}$. The ECG showed left ventricular hypertrophy with deeply inverted $\mathrm{T}$-waves in the left chest leads.

Cardiac catheterisation showed a left-to-right shunt at ventricular level with equal ventricular pressures. The catheter was advanced through the ductus arteriosus into the descending aorta where the systolic pressure was $35 \mathrm{~mm}$ lower, indicating ductal constriction.

During and after cardiac catheterisation, acidosis became increasingly severe despite administration of IV bicarbonate. The child was considered too ill to undertake surgery at this stage. In order to improve systemic perfusion which was largely dependent on patency of the ductus, prostaglandin E2 infusion at the rate of $0 \cdot 10 \mu \mathrm{g} / \mathrm{kg}$ per min was begun. This was delivered directly into the ductus from a catheter inserted via the umbilical artery. Within

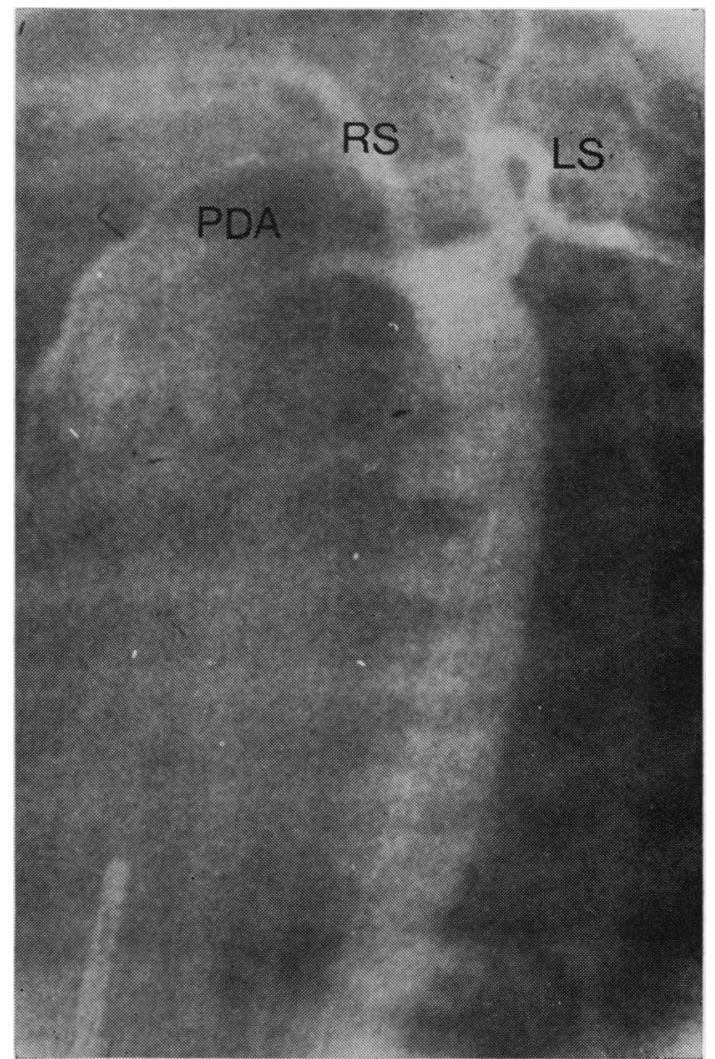

Fig. 3 Injection of contrast into the descending aorta, lateral projection. The left subclavian $(L S)$ and right subclavian $(R S)$ arteries arise from it and the narrowed ductus arteriosus $(P D A)$ fills.

15 min peripheral pulses became palpable and acidosis improved. The child remained anuric, however, and died on the operating table.

Necropsy showed situs solitus, normal connections, right-sided ascending aorta giving rise to both right and left common carotid arteries. The pulmonary artery was in continuity through a large ductus with a right-sided descending aorta from which arose the right and aberrant left subclavian arteries. The arch was grossly hypoplastic between the right common carotid and ductus. There was a large defect between the right ventricular infundibulum and the left ventricular outflow tract which appeared to be due to a complete absence of the infundibular septum.

\section{Discussion}

Patients with interruption of the aortic arch associated with a ventricular septal defect and persistent 
ductus arteriosus commonly present with heart failure in the first few days of life and $80 \%$ die in the first month. Therefore early recognition of the lesion is vital as surgical correction is possible (Tyson et al., 1970; Barratt-Boyes et al., 1972; Murphy et al., 1973; Losman et al., 1974; Trusler and Izukawa, 1975; Fishman et al., 1976; Ventemiglia et al., 1976; Monro et al., 1977). Origin of the right subclavian artery from the descending aorta has been reported with interruption of a left aortic arch (Evans, 1933; Kleinermann et al., 1958; Tabakin and Hanson, 1960, Zetterqvist, 1967; Morgan et al., 1970; Van Praagh et al., 1971; Subramanian, 1972; Kauff et al., 1973; Murphy et al., 1973; Trusler and Izukawa, 1975). We have been unable to find reports of an aberrant left subclavian artery associated with a hypoplastic right aortic arch.

At necropsy in infants with arch interruption the ductus arteriosus is usually widely patent with some wrinkling of the intima. Fournier and Zaidi (1960), Van Praagh et al. (1971), and Subramanian (1972) reported cases in which there had been significant narrowing of the ductus arteriosus. The combination of a right subclavian artery arising from the descending aorta and narrowing of the ductus arteriosus was present in the cases reported by Van Praagh et al. (1971), Subramanian (1972), and in 2 of our cases. In these, and in our 3rd case, a characteristic clinical picture of absent or markedly reduced limb pulses with bounding carotid artery pulsation was produced. This occurred because the subclavian arteries arose from the descending aorta, supplied by the narrowed ductus and by retrograde flow in the vertebral arteries. The detection of the large amplitude pulsation in the carotid arteries should suggest the clinical diagnosis. If carotid pulsation is not detected in an infant with heart failure, acidaemia, and absent limb pulses, aortic atresia-an inoperable lesion-may be mistakenly diagnosed. We have seen 2 patients with similar clinical findings caused by an intracranial arteriovenous fistula. The volume overload of the heart, due to the left-to-right intracranial shunt, caused heart failure and all the pulses were small except for the carotid arteries which were bounding as they supplied the arteriovenous fistula.

Infusion of prostaglandin E2 $0 \cdot 1 \mu \mathrm{g} / \mathrm{kg}$ per min has been used to dilate the ductus arteriosus in pulmonary atresia when pulmonary blood flow was dependent on its patency (Rudolph, 1977). Dilatation of the ductus by increasing lower limb flow helped to arrest the progressive acidosis accompanying ductal closure in one case. Aortic arch interruption is a further clinical situation where prostaglandin may be used. Mortality in this condition may thus be reduced as the infants' general condition at the start of surgery should be much improved by the adequate correction of acidosis.

\section{References}

Barratt-Boyes, B. G., Simpson, M., and Neutze, J. M. (1971). Intracardiac surgery on neonates and infants using deep hypothermia with surface cooling and limited cardiopulmonary bypass. Circulation, 43/44, Supplement 1, 25-30.

Barratt-Boyes, B. G., Nicholls, T. T., Brandt, P. W. T., and Neutze, J. M. (1972). Aortic arch interruption associated with patent ductus arteriosus, ventricular septal defect, and total anomalous pulmonary venous correction. Journal of Thoracic and Cardiovascular Surgery, 63, 367-373.

Celoria, G. C., and Patton, R. B. (1959). Congenital absence of the aortic arch. American Heart Journal, 58, 407-413.

Evans, W. (1933). Congenital stenosis (coarctation), atresia, and interruption of the aortic arch. Quarterly Journal of Medicine, 26, 1-31.

Fishman, N. H., Bronstein, M. H., Berman, W., Jr, Roe, R. B., Edmunds, L. H., Robinson, S. J., and Rudolph, A. M. (1976). Surgical management of severe aortic coarctation and interrupted arch in neonates. Journal of Thoracic and Cardiovascular Surgery, 71, 35-48.

Fournier, P., and Zaidi, Z. H. (1960). Congenital absence of the aortic arch. American Heart Journal, 59, 148-152.

Kauff, M. K., Bloch, J., and Baltaxe, H. A. (1973). Complete interruption of the aortic arch in adults. Radiology, 106, 53-57.

Kleinermann, J., Yang, W., Hackel, D., and Kaufman, N. (1958). Absence of the transverse aortic arch. Archives of Pathology, 65, 490-498.

Losman, J. G., Joffe, H. S., Beck, W., and Barnard, C. (1974). Successful total repair of interrupted aortic arch associated with ventricular septal defect and large patent ductus arteriosus. American Journal of Cardiology, 33, 566-571.

Monro, J. L., Brown, W., and Conway, N. (1977). Correction of type B interrupted aortic arch with ventricular septal defect in infancy. Journal of Thoracic and Cardiovascular Surgery, 74, 618-623.

Morgan, J. R., Forker, A. D., Fosburg, R. G., Neugebauer, M. K., Rogers, A. K., and Bemiller, C. R. (1970). Interruption of the aortic arch without a patent ductus arteriosus. Circulation, 42, 961-966.

Murphy, D. A., Lemire, G. G., Tessler, I., and Dunn, G. L. (1973). Correction of type B aortic arch interruption with ventricular and atrial septal defects in a three-day old infant. Journal of Thoracic and Cardiovascular Surgery, 65, 882-886.

Roberts, W. C., Morrow, A. G., and Braunwald, E. (1962). Complete interruption of the aortic arch. Circulation, 26, 39-59.

Rudolph, A. (1977). The ductus arteriosus. In Paediatric Cardiology, pp. 406-414. Edited by R. H. Anderson and E. A. Shinebourne. Churchill Livingstone: Edinburgh.

Subramanian, A. R. (1972). Coarctation and interruption of aorta proximal to origin of both subclavian arteries. Report of three cases presenting in infancy. British Heart Journal, 34, 1225.

Tabakin, B. S., and Hanson, J. S. (1960). Congenital absence of the aortic arch associated with patent ductus arteriosus and ventricular septal defect. American Journal of Cardiology, 6, 689-693.

Trusler, G. A., and Izukawa, T. (1975). Interrupted aortic arch and ventricular septal defect: direct repair through a median sternotomy incision in a 13-day old infant. Journal of Thoracic and Cardiovascular Surgery, 69, 126-131. 
Tyson, K. R. T., Harris, L. C., and Ngheim, Q. X. (1970). Repair of aortic arch interruption in the neonate. Surgery, 67, 1006-1010.

Van Praagh, R., Bernhard, W. F., Rosenthal, A., Parisi, L. F., and Fyler, D. C. (1971). Interrupted aortic arch: surgical treatment. American Journal of Cardiology, 27, 200-211.

Ventemiglia, R., Oglietti, J., Wukasch, D. C., Halliman, G. L., and Cooley, D. A. (1976). Interruption of the aortic arch. Surgical considerations. Journal of Thoracic and Cardiovascular Surgery, 72, 235-242.
Zetterqvist, P. (1967). Atypical coarctation of the aorta with bilateral vertebral-subclavian pathway. Scandinavian Journal of Thoracic and Cardiovascu!'ar Surgery, 1, 68-75.

Correspondence to J. L. Monro, FRCS, Southampton Western Hospital, Oakley Road, Southampton SO9 4WQ.

Received 18 April 1978

The following articles will appear in future issues of this journal:

Screening for cystic fibrosis in the newborn by meconium analysis. H. C. Ryley, L. M. Neale, T. D. Brogan, and P. T. Bray.

Medulloblastoma—a changing prognosis? N. McIntosh.

Foot length-a new and potentially useful measurement in the neonate. D. K. James, E. H. Dryburgh, and M. L. Chiswick.

Feeding the very low birthweight (1500 $\mathrm{g}$ and below) infant. One year's experience in an intensive care neonatal unit. I. Beddis and S. McKenzie.

A controlled trial of cyclophosphamide and azathioprine in Nigerian children with the nephrotic syndrome and poorly selective proteinuria. A. Adeniyi, R. G. Hendrickse, and J. F. Soothill.

Lomotil poisoning in children. J. A. Curtis and K. M. Goel.

Clinical evaluation and comparison of the Infrasonde, Arteriosonde, and mercury sphygmomanometer in measurement of blood pressure in children. J. M. Savage, M. J. Dillon, and J. F. N. Taylor.

Improved accuracy of continuous measurement of arterial oxygen tension in sick newborn infants: criteria for reading and recalibrating the electrode. A. R. Wilkinson, R. H. Phibbs, and G. A. Gregory. 